\title{
An Experimental Study of the Influence of Weak Convection on Perikinetic Coagulation
}

\author{
Z. W. Sun ${ }^{1}$ and R. L. Qiao \\ National Microgravity Laboratory, Institute of Mechanics, Chinese Academy of Sciences, Beijing, 100080 P. R. China
}

Received July 29, 1999; accepted November 23, 1999

\begin{abstract}
A remarkably increased coagulation rate for $2-\mu \mathrm{m}$ PS spheres was previously reported for a perikinetic coagulation experiment performed under microgravity conditions (1998, R. Folkersma, A. J . G. van Diemen, and H. N. Stein, J. Colloid Interface Sci. 206, 482); from this experiment, it was assumed that the leading factor slowing the coagulation process under normal gravitation was free convection due to gravity (1998, R. Folkersma, and H. N. Stein, J. Colloid Interface Sci. 206, 494). To test the influence of free convection as a single-effect factor on the coagulation process, a ground-based experiment was constructed. The coagulation rate of 2- $\mu \mathrm{m}$ PS spheres dispersed in water was determined by measuring the turbidity of the dispersion solution while convection-driven flows in the solution were checked with a visual magnification system. We found that it was possible to cease free convection-driven particle flows on the ground, as long as the experiments were carefully operated. The strength of convection was controlled by changing the temperature gradient applied to the sample cell. By monitoring both the coagulation rate and convection-driven flows simultaneously, our experiments showed that weak free convection (maximum speed $<150 \mu \mathrm{m} / \mathrm{s}$ ) actually has negligible effects on the coagulation rate. $\odot 2000$ Academic Press
\end{abstract}

Key Words: perikinetic; coagulation; convection; gravity.

\section{INTRODUCTION}

Folkersma et al. performed a series of experiments (1) on perikinetic coagulation under microgravity ( $\mu \mathrm{g}$ created by a sounding rocket), $1 g$, and high- $g$ conditions, and found that the coagulation rate under $\mu g$ is much higher than that under $1 g$. Their finding merits one's attention not only because it is unexpected by general theoretical prediction (3-8) and common sense, but also because the difference between the coagulation rates under gravitational influences and without gravitational influences is so significant. Folkersma et al. assume there are two aspects of gravity contributing to the difference: sedimentation and free convection. According to the data presented in their paper (2), the coagulation rate constants for $1 g$ (with a density difference between dispersions and liquid phase), $1 g$

\footnotetext{
${ }^{1}$ To whom correspondence should be addressed. E-mail: sunzw@ @c5.imech. ac.cn.
}

(density matched), and $\mu g$ are $0.754 \times 10^{-12}, 2.046 \times 10^{-12}$, and $8.616 \times 10^{-12}$, respectively. From these data we can see that the coagulation rate under $\mu g$ is 11.4 times larger than that under $1 g$ (density difference), in which the coagulation rate gain from the absence of sedimentation was $270 \%$ and the coagulation rate gain from the absence of free convection was $420 \%$. In other words, following their argument that free convection disrupts and therefore slows coagulation, if free convection is not present, then the coagulation rate should be $420 \%$ greater than that when free convection exists. This unusually large rate increase motivated us to test their argument.

Our experiment was based on the fact that it is possible to control and monitor the strength of free convection in aqueous polystyrene (PS) dispersions in a ground-based experiment while measuring the coagulation rate, and therefore we can test how free convection, as a single factor, affects the coagulation process on the ground. To make a reasonable comparison, we used the same sized $(2 \mu \mathrm{m})$ PS spheres as Folkersma et al., dispersed in water, and the same method as they used (measuring the turbidity of the sample solution) to determine the coagulation rate. We did indeed find that even a tiny temperature gradient was able to cause free convection in a dispersion solution. However, by carefully controlling the ambient temperature, it was possible to control, and even cease, free convection. When needed, convection flows were easily induced by applying a temperature gradient to the sample cell. Our concern is not shear-dominated coagulation, so our study is limited only to very weak convection (with the maximum speed $<150 \mu \mathrm{m} / \mathrm{s}$, probably microconvection is a more appropriate term to describe it), which is thought to be inevitable in normal ground-based experiments.

\section{EXPE RIMENTAL}

\section{Method}

Turbidity $(\tau)$ versus time $(t)$ was employed to monitor the saltinduced coagulation process of PS dispersions with an initial number concentration $N_{0}$, according to

$$
\left(1 / \tau_{0}\right)(d \tau / d t)_{t \rightarrow 0}=A\left(1 / N_{0}\right)(d N / d t),
$$

where $A$ is a constant (which depends on factors such as 


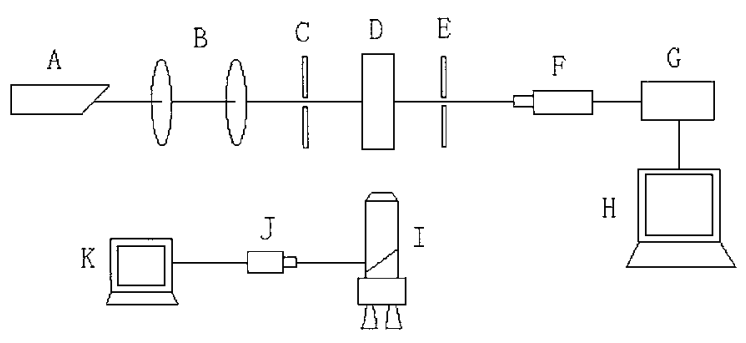

FIG. 1. Experimental setup: A, laser; B, lens group; C, aperture; D, cell; $\mathrm{E}$, aperture; F, detector; $\mathrm{G}$, data acquisition device and computer; $\mathrm{H}$, monitor; I, microscope; J, CCD camera; $\mathrm{K}$, monitor.

$d \ln \tau / d \lambda)$ and $\tau=(1 / L)\left(\ln T_{0} / T\right) . T, \lambda$, and $L$ are the transmission, the wavelength, and the optical path of light, respectively. The particles used, the detecting wavelength of light, and the particle concentration used were chosen to yield a $\tau / \tau_{0}$ vs $t$ curve for which the initial linear portion is sufficiently long and the slope is noticeable.

A schematic diagram of our experimental setup is shown in Fig. 1. A stabilized laser (the power fluctuation was less than $0.4 \%$ over $8 \mathrm{~h}$ ) was used as the detecting light source. The sample cell (object D) is the square-shaped container with interior dimensions of $10 \times 10 \times 43 \mathrm{~mm}(43 \mathrm{~mm}$ is its height, but in the most cases the height of sample column used in our experiment was only $10 \mathrm{~mm}$ ). The lens group (the two objects labeled B) was used to slightly enlarge the diameter of the laser beam in order to ensure that a greater population of PS particles passes through the detecting laser beam. One aperture (object C) was used to change the detecting section of the laser beam, and another aperture (object E) was used to protect the detector from unwanted scattered light, which could bring additional error (9). A microscope with a CCD camera and a monitor (the visual magnification power for the whole system was over 1000 times) were employed to monitor particle motion and the coagulation process. Figure 2 , curve $\mathrm{B}$, is a typical $\tau / \tau_{0} \sim t$ curve showing the rather long linear portion which is the key factor in our experiment.

Monodisperse PS latices (product of Duke Scientific Corp.) with diameters of $2.013 \pm 0.025 \mu \mathrm{m}$ were used in our experiment. The density of PS particles was $1.05 \mathrm{~g} / \mathrm{cm}^{3}$ at room temperature. $\mathrm{MgCl}_{2}$ was used as an electrolyte to induce rapid coagulation and after mixing with latex its concentration was $0.05 \mathrm{M}$. We used a $50 \% \mathrm{H}_{2} \mathrm{O}+50 \% \mathrm{D}_{2} \mathrm{O}$ mixture as the liquid phase whose density was well matched to the density of PS to reduce the effects of sedimentation and centrifugalization when convection flows exist. If the PS latex volume fraction is $1.5 \times 10^{-4}$, its number concentration $\left(N_{0}\right)$ is $3.4 \times 10^{7} \mathrm{~cm}^{-3}$. The theoretically estimated coagulation time $\left(T_{1 / 2}=3 \eta / 8 N_{0} K T\right)$ is about $3,000 \mathrm{~s}$.

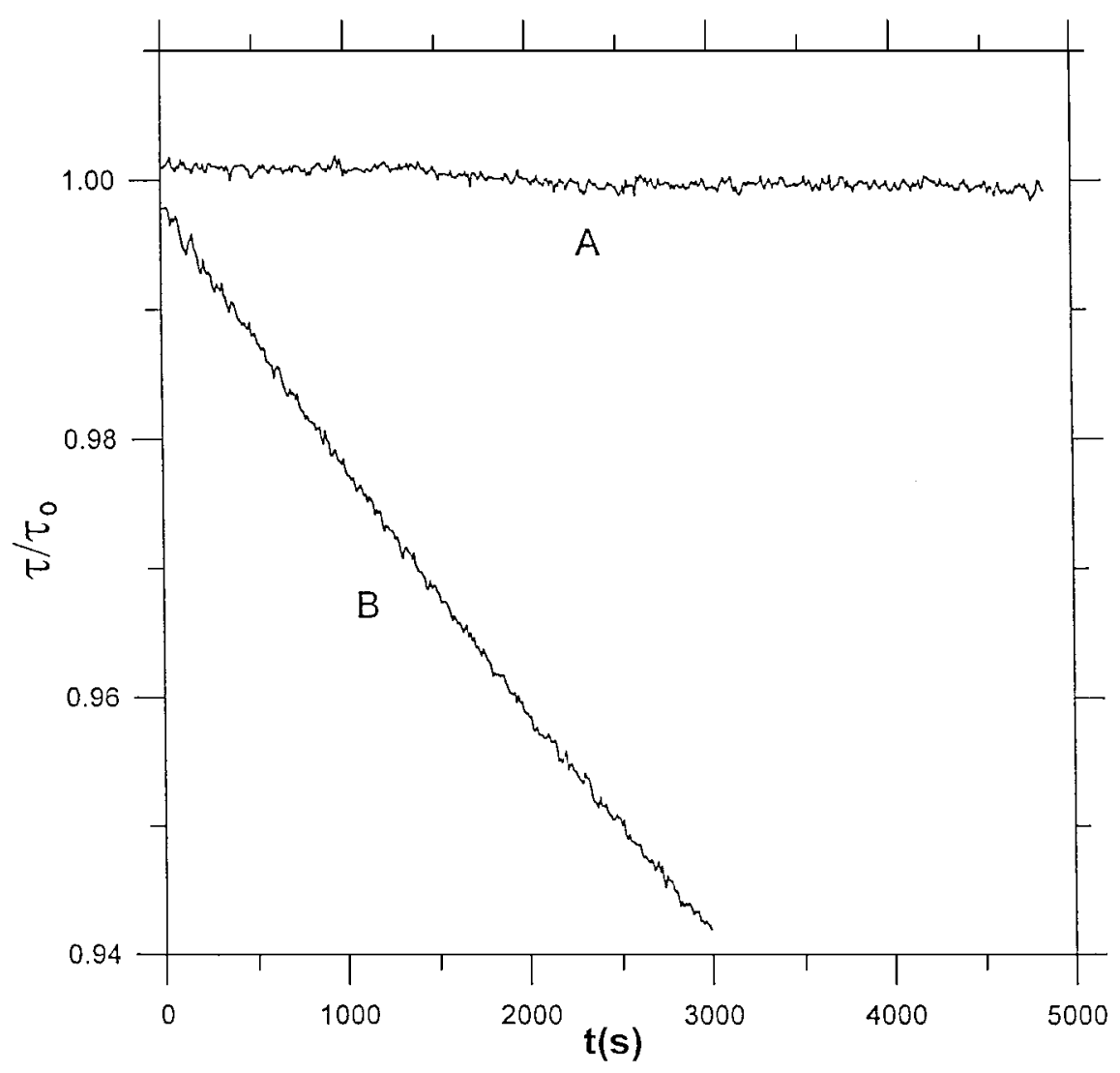

FIG . 2. Relative turbidity $\left(\tau / \tau_{0}\right)$ versus time $(t)$. (A) PS dispersion without electrolytes added; (B) PS dispersion with electrolytes added. 


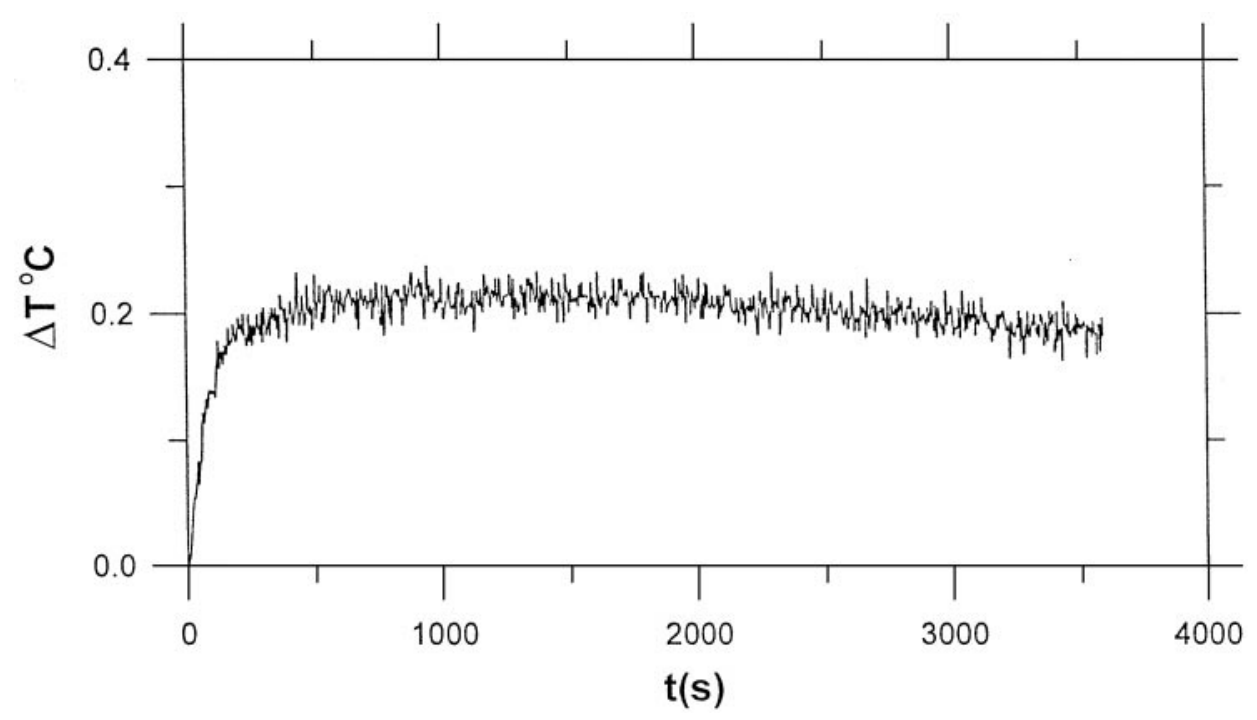

FIG . 3. Temperature difference versus time across the cell after a cool source was placed against one side wall.

Our visual magnification system was able to detect particles and their motions only, not the real convection of the liquid phase. We assume that if convection is not strong enough to cause particle motion, it will not contribute to coagulation; our definition for "convection" does not differentiate between the convection of the liquid and the convection of particles driven by the liquid's convection. More specifically, the term "convectiondriven particle flows" should be adopted to describe our definition of "convection", but for the sake of simplicity, we will use the term "convection" in most cases here. Therefore, when we write "no convection flows observed" in this paper, we mean that no directional particle motion driven by liquid convection was detected by our magnification system. This does not exclude the case that free convection was present, but too weak to move particles.

\section{Controlling Convection}

Convection flows were very sensitive to changes in the surrounding temperature. For example, flows were easily "turned on" by people walking toward the sample, and the breeze produced by the air-conditioner could easily cause convection flows with speeds of $40 \mu \mathrm{m} / \mathrm{s}$. In order to "turn off" the flows, PS latex, water, and electrolytes were kept at the same temperature as the surroundings before they were mixed for experiments. Flows in the solution would start right after mixing, but would then cease within $30 \mathrm{~s}$ to $1 \mathrm{~min}$ later if the ingredients were at the same temperature and there was no change in the surrounding temperature. Thankfully, our $\tau / \tau_{0} \sim t$ curve has a long linear portion, and only the slope of that portion is of any importance.

To initiate convection flows, we simply placed a cell with relatively cool water close to one of the side walls of the sample cell. The strength of the convection flow could be changed by adjusting the temperature of the cooler cell or changing its distance from the sample cell. This simple method was sufficient to main- tain convection flows going. Figure 3 shows the progression of the temperature difference $\left(\Delta T,{ }^{\circ} \mathrm{C}\right)$ versus time $(t)$ between the cooler wall and its opposite wall, both of which were measured with thermoelectric couplers from inside the sample cell.

Actually, it is much easier to induce the convection flows than to avoid them on a ground-based experiment. We found that even a $0.1{ }^{\circ} \mathrm{C}$ temperature difference may induce particles' motion with maximum speeds of up to $15 \mu \mathrm{m} / \mathrm{s}$. Our equipment was effective only for monitoring particles' motions near the side wall where higher particle speeds are found.

\section{Validity Test}

Before the experiments, we tested the validity of using turbidity versus time (as discussed above) for determining the coagulation rate. First, as a blank test, we tested a dispersion solution (only PS particles dispersed in liquid phase) with no electrolytes added; curve A in Fig. 2 shows its " $\tau / \tau_{0} \sim t$ " behavior, which is flat for a quite long period of time (over $1 \mathrm{~h}$ ), meaning a slope of zero, or that there was no coagulation occurring. It also shows that the influence of particles' sedimentation on $\tau / \tau_{0}$ is negligible during the experimental process (the estimated sedimentation rate for our $2-\mu \mathrm{m}$ PS spheres was about $0.1 \mu \mathrm{m} / \mathrm{s}$ ).

Second, we tested the linear behavior of the $\tau / \tau_{0} \sim t$ data during the coagulation process. Curve B in Fig. 2 corresponds to the dispersion solution when $\mathrm{MgCl}_{2}$ was added. Its linear portion is quite long; this is particularly important for our current study because we can therefore discount the data which might be affected by the initial random flows without losing the correct slope of the linear part of the data, the slope being of interest.

Third, we tested whether the slope of $\tau / \tau_{0} \sim t$ corresponds to the coagulation rate. Since the coagulation rate is supposed to be proportional to the initial number concentration $N_{0}$ of dispersed particles, we can determine the coagulation rate of different turbidity measurements for different $N_{0}$. Figure 4 shows a 


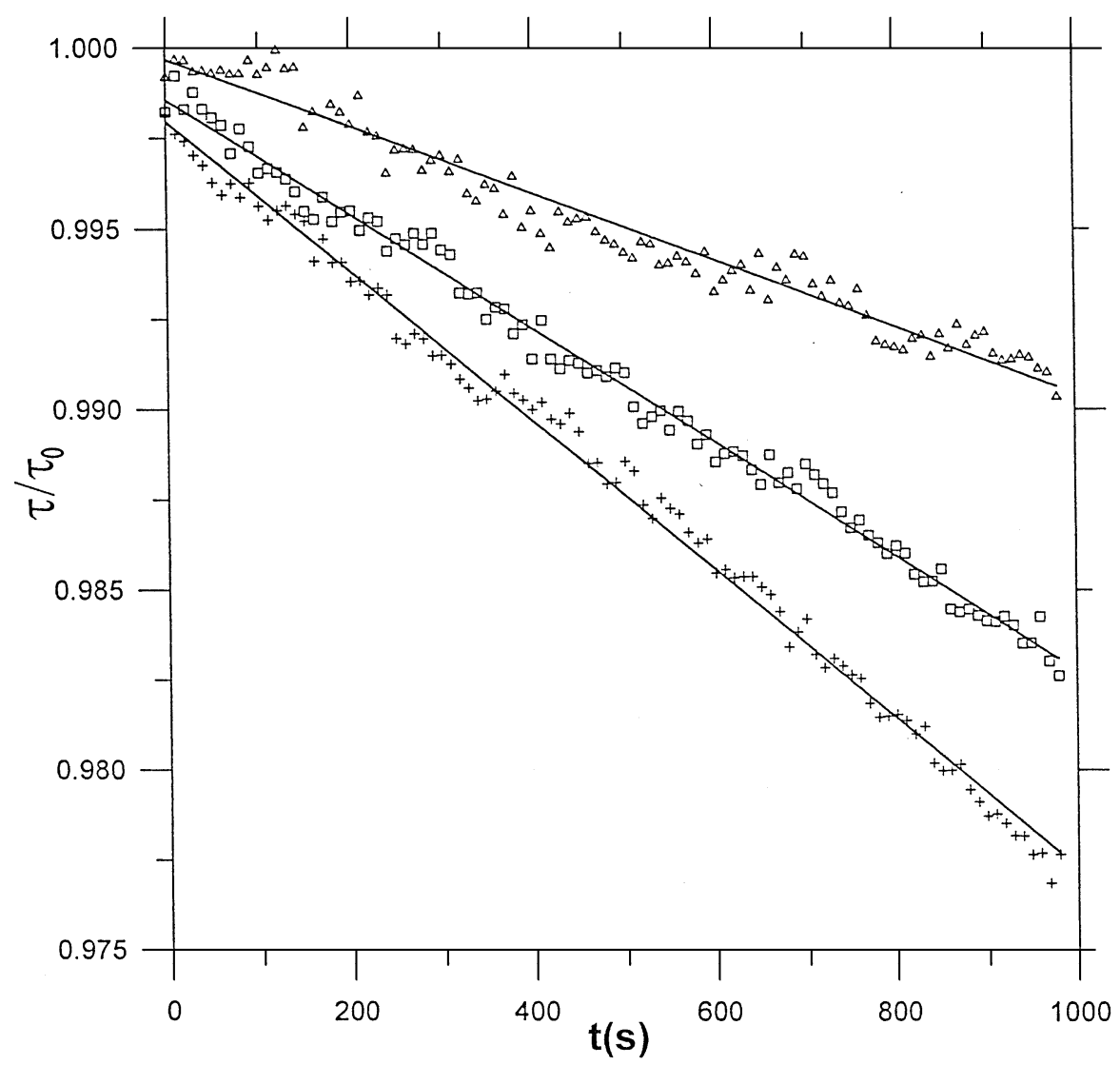

FIG . 4. Relative turbidity $\left(\tau / \tau_{0}\right)$ versus time $(t)$ for dispersed PS particles with different initial concentrations. The three lines represent solutions having an initial particle concentration ratio of $(+) 1,(\square) 0.8$, and $(\triangle)$ 0.5. The ratio of the coagulation rates for these samples was calculated to be 1:0.79:0.47.

group of $\tau / \tau_{0} \sim t$ curves for three samples having initial particle concentration ratios of 1:0.8:0.5, respectively. According to the slopes of the lines made by linearly fitting the data, the ratio of the coagulation rates for these samples can be calculated to be 1:0.79:0.47, which is in agreement with the coagulation rates, as we had assumed.

Apparently, our experimental procedure is valid only for comparing relative coagulation rates but that is enough as long as our concern is whether weak convection would make a significant difference to the perikinetic coagulation process.

\section{RESULTS AND DISCUSSION}

If a temperature gradient is not applied to the sample, convection flows are weak (generally, less than $50 \mu \mathrm{m} / \mathrm{s}$ ). So, first, we kept the convection flows at a low level (15 to $20 \mu \mathrm{m} / \mathrm{s}$ ). Two parallel coagulation experiments were done separately under the same initial conditions: PS latex volume fraction $1.5 \times 10^{-4}$, and $0.05 \mathrm{M} \mathrm{MgCl}_{2}$ in the final solution. Figure 5 shows $\tau / \tau_{0} \sim t$ curves for the two experiments: the squares correspond to the coagulation process without convection flows, and the crosses correspond to the coagulation process with convection flows "turned on" (convection flows with maximum speeds of approximately
$20 \mu \mathrm{m} / \mathrm{s}$ ). The difference in the slopes (which are proportional to the coagulation rates) of these two fitted lines is well within the reproducibility of our measurements $(5 \%)$. We can see that free convection flows do not bring noticeable changes to the coagulation process. When stronger convection flows (maximum speeds of $100 \mu \mathrm{m} / \mathrm{s}$ ) were imposed, there was still no significant influence on the coagulation rate observed.

To check whether the change in $\tau / \tau_{0}$ was related solely to the change in the coagulation rate and not affected by convection flows, we did a separate "blank" experiment with only PS particles in solution. No electrolytes were added, and therefore no coagulation was expected even when convection flows were "turned on". The data $\left(\tau / \tau_{0} \sim t\right)$ are shown in curve A of Fig. 2. There was no noticeable change (except fluctuations) in $\tau / \tau_{0}$ when the strengths of convection flows were altered in a range of maximum speeds from 0 to $150 \mu \mathrm{m} / \mathrm{s}$.

Curve B in Fig. 6 represents the $\left(\tau / \tau_{0} \sim t\right)$ coagulation process when we imposed the convection flows (with a maximum speed of approximately $150 \mu \mathrm{m} / \mathrm{s}$ ) in the solution. Curve $\mathrm{A}$ is for its contrast experiment in which no convection flow was imposed. We can see that at the beginning stage, both curves coincide well, and then gradually separate. Apparently, when larger particles become appreciable, the shear due to convection plays an increasingly important role. 


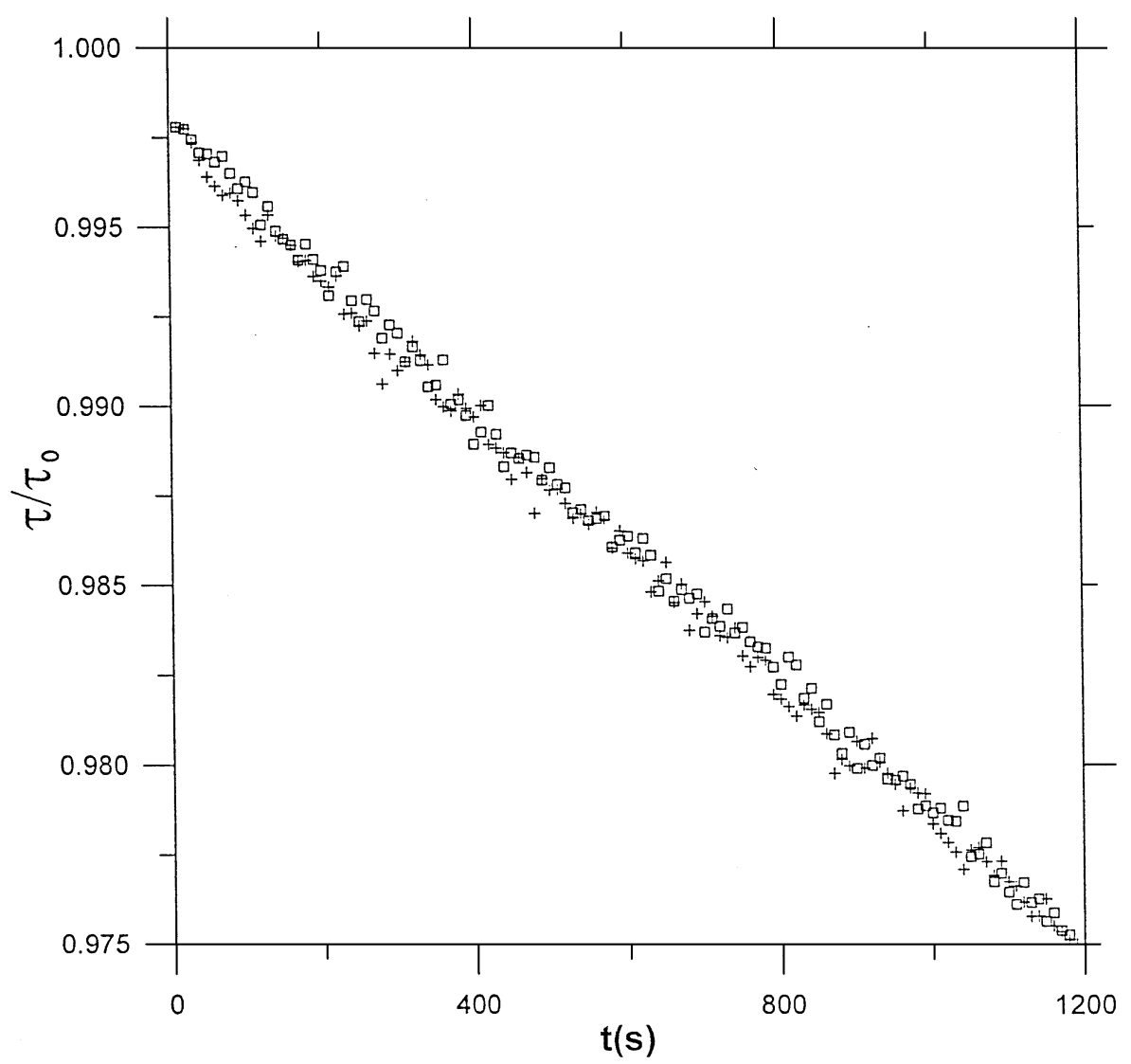

FIG . 5. Relative turbidity $\left(\tau / \tau_{0}\right)$ versus time $(t)$ for solutions with convection flows with a maximum speed of $20 \mu \mathrm{m} / \mathrm{s}(+)$ and without convection flows $(\square)$.

Our observation does not support the assumption that free convection is a main factor that profoundly slows the perikinetic coagulation process due to the disruption of aggregates by convection-driven flows on a ground-based experiment. This investigation was limited only to very weak convection since it is perikinetic coagulation, which is of concern, and convection flows stronger than those that we imposed during the experiment are easy to prevent on the ground. Actually, there has been a lot of research work devoted to the influence of strong convection on the coagulation process; for example, see (10).

The shear due to convection may disrupt aggregates and therefore retard the coagulation process if primary particles in aggregates are loosely bonded as a result of reversible aggregation occurring at the second minimum of the potential surface of particles. As explained in (2), however, this is possible only when the attractive interaction force between two particles is low (because of surface roughness of the particles). However, this did not seem to occur in our study. More likely, the coagulation in our experiments occurred in an irreversible process in the deep, primary minimum. So the characteristics of the PS particles we used might be different from those in Folkersma's experiment.

However, it is somewhat puzzling that our preliminary experiments showed that the coagulation for $2-\mu \mathrm{m}$ PS spheres dis- persed in density-matched liquid was about $11 \%$ faster than that in density-unmatched liquid (compared with a $220 \%$ difference in Folkersma's experiment). If the coagulation happened only at the primary minimum, we have no proper explanation for this fact.

Our experimental procedure was quite different from Folkersma's procedure. For example, the same sample was used repeatedly in Folkersma's experiments, with the aggregates being broken down by ultrasonic vibration between experiments. Their approach ensures that the PS concentration is unchanged in a series experiments. But we used fresh samples in each experiment. Actually, the application of ultrasonic vibration to break down aggregates would make it impossible to stop convection flows within a short time period for a ground-based experiment. The ultrasonic energy raises the temperature of the sample and therefore the sample would need more time to reach a temperature equilibrium with its surroundings before convection can cease. Actually, we did try using ultrasonic vibration to break down aggregates, but we found that the original level of the transmission signal could not be regained. This shows that aggregates in our experiments were more closely bonded.

Furthermore, as mentioned in (1), even if the aggregates were bonded at the second minimum, the shear due to convection plays 


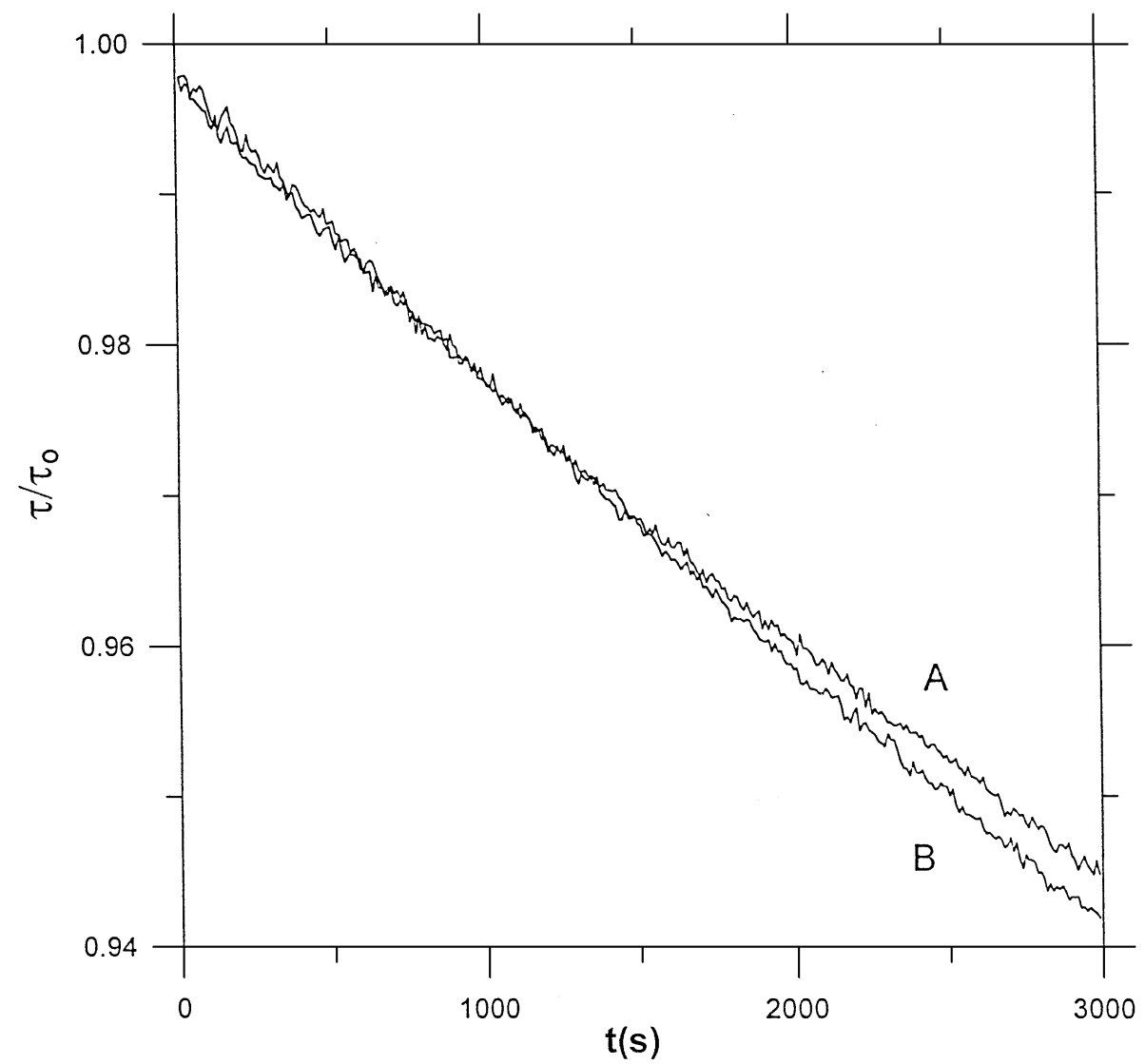

FIG . 6. Relative turbidity $\left(\tau / \tau_{0}\right)$ versus time ( $t$ ) for solutions with convection flows with a maximum speed of $150 \mu \mathrm{m} / \mathrm{s}$ (B) and without convection flows (A).

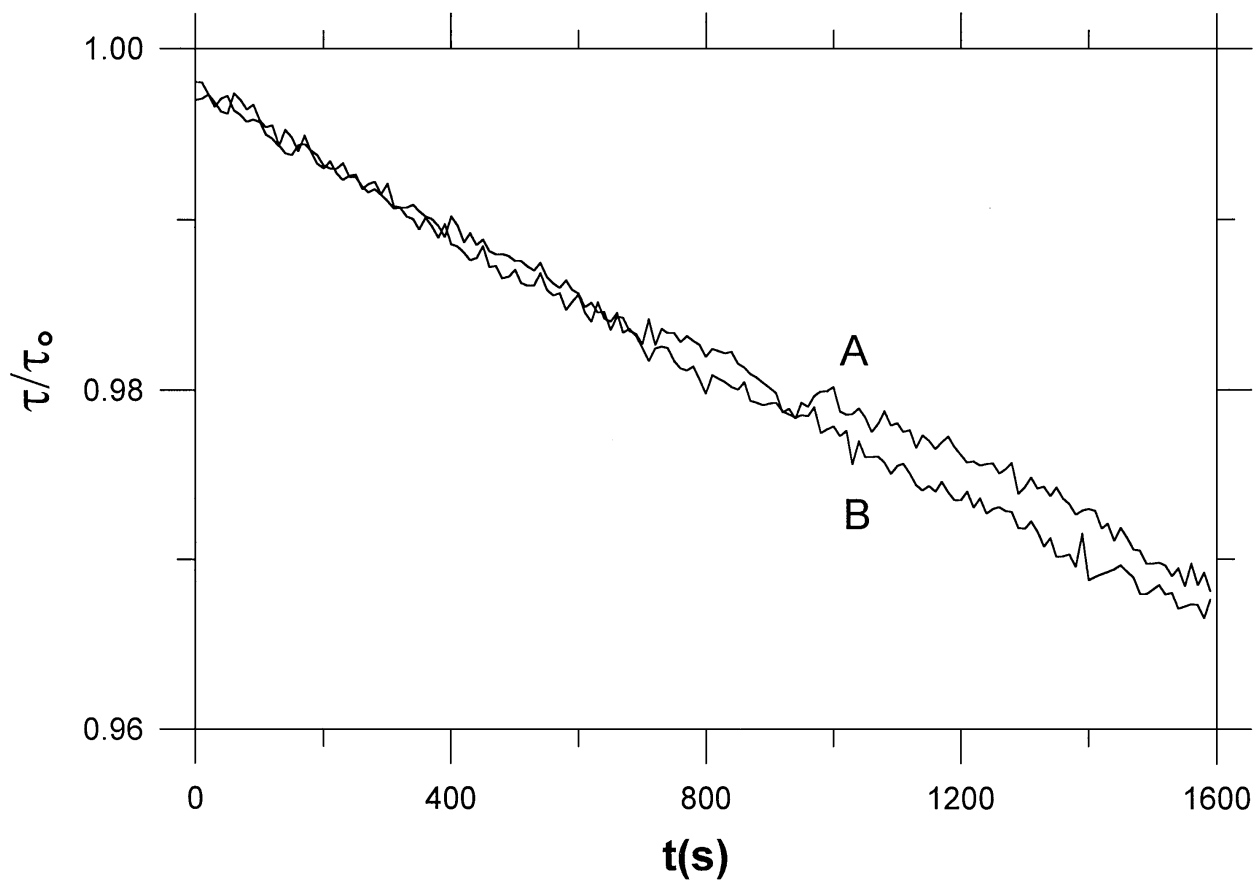

FIG. 7. Relative turbidity $\left(\tau / \tau_{0}\right)$ versus time $(t)$ for solutions with $\mathrm{NaCl}$ as electrolyte $(\mathrm{A})$ and $\mathrm{MgCl}_{2}$ as electrolyte. 
two counteracting roles in the coagulation process: causing more collisions between particles and disrupting formed aggregates. Accelerating or retarding coagulation will depend on which role predominates. Particle concentration, shear rate, and the depth of the second minimum would make a difference.

In all our experiments described above we used $\mathrm{MgCl}_{2}$ to induce rapid coagulation because it is much more efficient than 1:1 electrolyte, $\mathrm{NaCl}$. Using less electrolyte would cause less change in density (and viscosity also) of the final solution, which is desirable because we need a larger difference in density for density-matched and unmatched experiments to test the sedimentation influence. However, there is a question raised naturally: what could happen if $0.5 \mathrm{M} \mathrm{NaCl}$ solution was used to replace $\mathrm{MgCl}_{2}$ in our experiments, because it may change the nature of the particles' interaction $\left(0.05 \mathrm{M}\right.$ for $\mathrm{MgCl}_{2}$ may be a little too high relative to its critical coagulation concentration as opposed to $0.5 \mathrm{M}$ for $\mathrm{NaCl}$ ). We redid our coagulation experiment with $0.5 \mathrm{M} \mathrm{NaCl}$ (in final) solution instead of $0.05 \mathrm{M}$ $\mathrm{MgCl}_{2}$. Curves $\left(\tau / \tau_{0} \sim t\right)$ representing the coagulation process in both $0.5 \mathrm{M} \mathrm{NaCl}$ (in final) solution (A) and $0.05 \mathrm{M} \mathrm{MgCl}_{2}$ solution (B) are showed in Fig. 7. There were no special measures taken to control free convection flows in both cases. We can see that the coagulation rates for both cases are close. From linear fitting, the difference between the slopes of curves A and B is less than $8 \%$. Taking experimental inaccuracy and the changes in viscosity, density, etc. into account, the difference in the slopes of the curves is actually negligible. From this comparison we confirmed that using $0.5 \mathrm{M} \mathrm{NaCl}$ or $0.05 \mathrm{M} \mathrm{MgCl}_{2}$ as electrolyte in our coagulation experiment (when free convection exists) does not make a noticeable difference. On the other hand, our previous experiments have shown that the coagulation rate in $0.05 \mathrm{M}$ $\mathrm{MgCl}_{2}$ solution does not change significantly whether a (weak) convection flows exist or not. If convection flows can significantly reduce the coagulation rate when $0.5 \mathrm{M} \mathrm{NaCl}$ is used instead of $0.05 \mathrm{M} \mathrm{MgCl}_{2}$, we should have observed a much lower rate than that for the $0.05 \mathrm{M} \mathrm{MgCl}_{2}$ experiment. But this is not the case. A reasonable inference based on this fact is that weak free convection-driven flows do not noticeably contribute to perikinetic coagulation for $2-\mu \mathrm{m}$ particles' dispersion even when $0.5 \mathrm{M} \mathrm{NaCl}$ is used as electrolyte.

\section{CONCLUSION}

An experimental setup was constructed to make it possible to separately test how convection-driven flows affect the perikinetic coagulation process in a ground-based experiment. Our experiment provided direct evidence showing that weak free convection-driven flows do not noticeably contribute to perikinetic coagulation for $2-\mu \mathrm{m}$ particles' dispersion. Our observations show that free convection, as a main cause, profoundly slowing the perikinetic coagulation process is, at least, not the general case. We assume that differences in the characteristics of the different PS particles and experimental methods used in Folkersma's experiment and ours might account for the different results.

\section{ACKNOWLEDGMENTS}

This work was partially supported by Grant 95-Yu-34 from the Ministry of Science and Technology and Grant 19789201 from the National Natural Science Foundation of China.

\section{REFERENCES}

1. Folkersma, R., van Diemen, A. J. G., and Stein, H. N., J. Colloid Interface Sci. 206, 482 (1998).

2. Folkersma, R., and Stein, H. N., J. Colloid Interface Sci. 206, 494 (1998).

3. Melik, D. H., and Fogler, H. S., J. Colloid Interface Sci. 101, 84 (1984).

4. Wang, Y. G., and Wen, C. S., J. Fluid Mech. 214, 599 (1990).

5. Zinchenko, A. Z., and Davis, R. H., J. Fluid Mech. 280, 119 (1994).

6. Ven, T. G. M. van de, and Mason, S. G., Colloid Polym. Sci. 255, 794 (1977).

7. Logtenberg, E. H. P., and Stein, H. N., J. Colloid Interface Sci. 104, 258 (1984).

8. Qiao, R. L., and Wen, C. S., J. Colloid Interface Sci. 178, 364 (1996).

9. Ferri, F., Bassini, A., and Paganini, E., Appl. Opt. 36 (4), 885 (1997).

10. Russel, W. D., Saville, D. A., Schowalter, W. R., "Colloidal Dispersions," Chapter 8, Cambridge Univ. Press, Cambridge, 1989. 